\title{
Energy resolved electrochemical impedance spectroscopy for electronic structure mapping in organic semiconductors
}

\author{
V. Nádaždy, ${ }^{1, a)}$ F. Schauer, ${ }^{2,3}$ and K. Gmucová ${ }^{1}$ \\ ${ }^{1}$ Institute of Physics SAS, Dúbravská cesta 9, 84511 Bratislava, Slovak Republic \\ ${ }^{2}$ Faculty of Education, Trnava University in Trnava, 91843 Trnava, Slovak Republic \\ ${ }^{3}$ Faculty of Applied Informatics, Tomas Bata University in Zlin, 76005 Zlin, Czech Republic
}

(Received 23 June 2014; accepted 2 October 2014; published online 10 October 2014)

\begin{abstract}
We introduce an energy resolved electrochemical impedance spectroscopy method to map the electronic density of states (DOS) in organic semiconductor materials. The method consists in measurement of the charge transfer resistance of a semiconductor/electrolyte interface at a frequency where the redox reactions determine the real component of the impedance. The charge transfer resistance value provides direct information about the electronic DOS at the energy given by the electrochemical potential of the electrolyte, which can be adjusted using an external voltage. A simple theory for experimental data evaluation is proposed, along with an explanation of the corresponding experimental conditions. The method allows mapping over unprecedentedly wide energy and DOS ranges. Also, important DOS parameters can be determined directly from the raw experimental data without the lengthy analysis required in other techniques. The potential of the proposed method is illustrated by tracing weak bond defect states induced by ultraviolet treatment above the highest occupied molecular orbital in a prototypical $\sigma$-conjugated polymer, poly[methyl(phenyl)silylene]. The results agree well with those of our previous DOS reconstruction by post-transient space-charge-limited-current spectroscopy, which was, however, limited to a narrow energy range. In addition, good agreement of the DOS values measured on two common $\pi$-conjugated organic polymer semiconductors, polyphenylene vinylene and poly(3-hexylthiophene), with the rather rare previously published data demonstrate the accuracy of the proposed method. (C) 2014 AIP Publishing LLC. [http://dx.doi.org/10.1063/1.4898068]
\end{abstract}

Determination of the electronic structures of organic semiconductors has major relevance for studies of charge/ energy transport and recombination phenomena in organic electronics. However, weak molecular coupling and disordered structures often preclude application of the spectroscopic methods used for inorganic semiconductors. ${ }^{1}$ Electrochemical spectroscopic methods tend to fill this gap. Electrochemical impedance spectroscopy (EIS) has been known for decades and has served many purposes, from studies of electrochemical reaction mechanisms to investigations of passive surfaces. ${ }^{2}$ EIS development has also been positively influenced by activities related to clarification of solid-electrolyte processes over the last 40 to 50 years. ${ }^{3}$ A cumulative paper describing progress in the examination of various nanostructured and organic materials by EIS was published by Bisquert et al. ${ }^{4}$ Additionally, several other electrochemical methods exist for study of electronic structures in organic semiconductors, based on direct determination of the density of states (DOS) at the Fermi energy., Electrochemical cyclic voltammetry (CV) of conducting polymers and molecular solid films has also been interpreted in terms of the electronic DOS. ${ }^{7}$ The $\mathrm{CV}$ of organic films is generally characterized by a broad non-Nernstian signal, which is interpreted as an indication of the underlying Gaussian DOS that is common in disordered organic materials. Recently, CV analysis has been improved by

\footnotetext{
${ }^{\text {a) }}$ Author to whom correspondence should be addressed. Electronic mail: nadazdy@savba.sk
}

including the formation of both polarons and bipolarons and taking their respective DOS values into account. ${ }^{8}$ Other techniques use organic thin-film transistor structures to derive the DOS by Kelvin probe force microscopy. ${ }^{9}$

In this letter, we present an energy resolved EIS (EREIS) method based on the interaction between an organic semiconductor and an electrolyte at an interface via a oxidation/reduction (redox) reaction. Measurement of the charge transfer resistance via the redox reaction gives direct information about the DOS in organic semiconductors. The power of this method is illustrated by tracing the defect states from weak bonds (WB) above the highest occupied molecular orbital (HOMO) induced by ultraviolet (UV) treatment of a prototypical $\sigma$-conjugated polymer, poly[methyl(phenyl)silylene] (PMPSi). These results agree well with those of our previous DOS reconstruction by post-transient space-chargelimited-current spectroscopy, ${ }^{10,11}$ although it is limited to a narrow energy range. Good agreement between the DOS values measured for two common $\pi$-conjugated organic polymer semiconductors, polyphenylene vinylene (PPV) and poly(3-hexylthiophene) (P3HT), and the rather rare previously published data ${ }^{5,12}$ provide further proof of the accuracy of the proposed method.

The EIS method is based on interaction between a thin organic film and an electrolyte via redox reactions in an electrochemical cell (Fig. 1). This interaction is similar to extraction of an electron by an acceptor and capture of an electron by a donor at a semiconductor surface through common solid state reactions. The charge-transfer current density $j$ between 


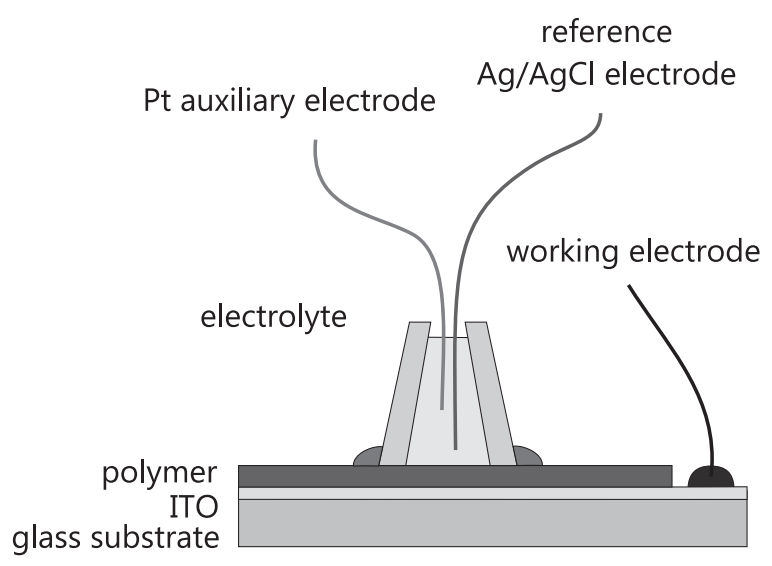

FIG. 1. Sample arrangement of ER-EIS method: The electrochemical cell is placed on the conductive glass substrate with the deposited polymer film that acts as the working electrode. Pt wire serves as the auxiliary electrode, and $\mathrm{Ag} / \mathrm{AgCl}$ acts as the reference electrode.

the semiconductor surface with electron concentration $n_{\mathrm{s}}$ and the electrolyte with concentration $[A]$ of the redox (donor/ acceptor) pair is ${ }^{13}$

$$
j=e k_{e t} n_{s}[A]
$$

where, $e$ is the elemental charge and $k_{e t}$ is the charge-transfer coefficient in the $10^{-17}-10^{-16} \mathrm{~cm}^{4} \mathrm{~s}^{-1}$ range. ${ }^{3}$ The semiconductor surface electron concentration $n_{s}$ is a variable that can be measured and controlled experimentally by varying the semiconductor surface potential. If the concentration of surface states of the organic film is negligible, then information about the electron states of the organic film at the energy position corresponding to the instantaneous electrochemical electrolyte potential can be obtained directly. A low surface state concentration can be achieved by preparing the film in an inert atmosphere. Various elementary processes of the semiconductor/electrolyte system respond to system perturbations at different rates and the corresponding relaxation processes become dominant in impedance spectra measured at various frequencies $\omega$. Therefore, the individual components of these processes can be identified and discriminated via impedance measurements over a specific frequency range. ${ }^{14}$

Using Eq. (1), we can express the DOS function in the semiconductor at the Fermi energy $g\left(E_{F}\right)$ in terms of the charge transfer resistance $R_{c t}(U)$ measured under applied voltage $U$ as

$$
g\left(E_{F}=e U\right)=\frac{\mathrm{d} n_{s}}{\mathrm{~d}(e U)}=\frac{1}{e k_{e t}[A] S} \frac{\mathrm{d}(j S)}{\mathrm{d}(U)}=\frac{1}{e k_{e t}[A] S R_{c t}} .
$$

We can experimentally access the charge transfer resistance $R_{c t}=\mathrm{d} U / \mathrm{d}(j S)$ using the superimposed perturbation $\mathrm{d} U$ at a suitable frequency $\omega$ (Ref. 14) under applied voltage $U$, where $S$ is the sample area. The desired DOS function $g(E)$ can be derived directly from the measured charge transfer resistance $R_{c t}(U)$ at the instantaneous position of the Fermi energy given by the applied potential $E_{F}=e U$ in Eq. (2). ${ }^{15}$

The ER-EIS technique has three unique advantages: (i) the diffusion character of the charge-transfer current density (see Eq. (1)) and its consequent independence of the results for any transport quantity, e.g., the mobility and its dependence on the applied field; ${ }^{16}$ (ii) the ability to control shifts in the semiconductor Fermi level position using the external voltage $U$, provided that the voltage shift of the electrolyte chemical potential is neglected (this is justified by the electrolyte's much larger DOS value); and (iii) the linearity of the electrochemical systems, ${ }^{1}$ which enable application of the perturbation method.

The measurements were performed using a common three-electrode electrochemical cell with volume of about $200 \mu \mathrm{l}$ (Fig. 1). The polymer film spin-coated on the indium tin oxide (ITO) substrate acted as the working electrode, while the $\mathrm{Ag} / \mathrm{AgCl}$ and $\mathrm{Pt}$ wires were the reference and auxiliary electrodes, respectively. The ER-EIS method probes the organic semiconductor via the interface between semiconductor and electrolyte. Because both the organic materials under study and the electrolyte are highly sensitive to the ambient atmosphere, an inert atmosphere is required to prevent degradation of the components. A glove box with protective $\mathrm{N}_{2}$ atmosphere (oxygen and moisture below $20 \mathrm{ppm}$ and $2 \mathrm{ppm}$, respectively) was used in all experimental steps, including sample preparation, manipulation and/or treatment, and measurement. The film potential with respect to the reference electrode was controlled by a potentiostat. The electrolyte solution used was a $0.1 \mathrm{M}$ solution of tetrabutylammonium hexafluorophosphate $\left(\mathrm{TBAPF}_{6}\right)$ in acetonitrile, allowing a voltage (energy) window of $\pm 3 \mathrm{~V}( \pm 3 \mathrm{eV})$ with respect to the $\mathrm{Ag} /$ $\mathrm{AgCl}$ reference electrode (vacuum). This range covers most of the band gaps of common organic semiconductors. Impedance measurements were performed using an impedance/gain-phase analyzer (Solartron Analytical, model 1260) in a frequency range of $0.1-10 \mathrm{~Hz}$ with $\mathrm{AC}$ voltage amplitude of $100 \mathrm{mV}$, and a DC voltage ramp sweep rate of $10 \mathrm{mV} / \mathrm{s}$. One common feature of all electrochemical methods is the possibility of irreversible electrolyte degradation. In particular, reaction product accumulation on the electrode, adsorption of solution impurities, and oxide layer growth may occur. To avoid these problems, each ER-EIS measurement was split in two separate sweeps. Both sweeps started at zero bias voltage, with one sweep being made to positive potentials and the other made to negative potentials. The final ER-EIS spectrum was then formed by joining the positive and negative branches. The error of this procedure can be neglected because it follows on from a systematically-performed reproducibility check.

The error of the method can be discussed in terms of the independent and dependent variables in the measured DOS plot. The energy position error is controlled by the precision of the semiconductor surface potential measurement, which is influenced by the electrolyte resistance and the amplitude of the perturbation $\mathrm{d} U$. The influence of the electrolyte resistance is eliminated by the three-electrode configuration, and thus, the voltage (energy) error is determined solely by $\mathrm{d} U(\mathrm{~d} e U)$, and is $\pm 50 \mathrm{mV}( \pm 50 \mathrm{meV})$ in our case. The DOS value error is determined by the impedance measurement. The bridge impedance analyzer used allowed measurement precision of $5 \%$ in all ranges, which produces a uniform DOS value error.

The ER-EIS method's performance was tested on three common polymers. PMPSi polymer films were spin-coated 
on ITO coated glass from a 0.5 wt. \% solution of PMPSi in tetrahydrofuran (THF) at $900 \mathrm{rpm}$ for $60 \mathrm{~s}$, followed by drying at $60^{\circ} \mathrm{C}$ for $3 \mathrm{~h}$. UV treatment was performed using a $345 \mathrm{~nm}$ wavelength and $1 \mathrm{mWcm}^{-2}$ power UV laser diode. Poly[2-methoxy-5-( $3^{\prime}, 7^{\prime}$-dimethyloctyloxy)-1,4-phenylenevinylene] (MDMO-PPV) and P3HT films were spin-coated from $0.5 \mathrm{wt}$. \% solutions in $\mathrm{CHCl}_{3}$ at $1800 \mathrm{rpm}$ for $40 \mathrm{~s}$, followed by annealing at $110^{\circ} \mathrm{C}$ for $5 \mathrm{~min}$. Before spin coating, all solutions were filtered using a $200 \mathrm{~nm}$ polytetrafluoroethylene (PTFE) filter. The films were approximately $100 \mathrm{~nm}$ thick.

The electronic structure in terms of DOS function, $g(E)$, was measured in all three polymers, including the deep states, which are important for recombination, and the shallow HOMO and LUMO (lowest unoccupied molecular orbital) states, which are important for charge transport. ${ }^{1}$ The energy scale was recalculated from the applied bias voltage to $\mathrm{eV}$ using the energy $-4.66 \mathrm{eV}$ of the $\mathrm{Ag} / \mathrm{AgCl}$ reference electrode. The energy scale is thus directly related to the zero vacuum energy level.

The extreme sensitivity of the ER-EIS method is demonstrated by DOS measurements of PMPSi films prepared from THF solutions under various conditions (Fig. 2). Here, line 1 depicts the DOS of a PMPSi film spin-coated from a filtered PTFE solution and subsequently bubbled for $20 \mathrm{~min}$ with Ar, while line 2 indicates a PMPSi film spin-coated from the solution without initial filtering or bubbling. Line 3 indicates a film spin-coated from the filtered but not bubbled solution, while line 4 shows the DOS for a film prepared from the filtered and bubbled PMPSi solution that was spin-coated and dried in air. Line 1 shows the HOMO at $5.89 \mathrm{eV}$ and the LUMO at $1.80 \mathrm{eV}$ with Gaussian distributions $\left(\sigma_{\mathrm{HOMO}}=0.48 \mathrm{eV}, \sigma_{\mathrm{LUMO}}=0.29 \mathrm{eV}\right)$. The polaron band gap has a value of $4.09 \mathrm{eV}$ in accordance with the literature. ${ }^{17}$ This DOS spectrum implies hopping charge transport via the Gaussian distributed localized states to be the predominant transport path. ${ }^{16,18}$ There is also an absence of deep traps in the band gap between the HOMO and LUMO, where the DOS is $4-5$ orders of magnitude lower than the

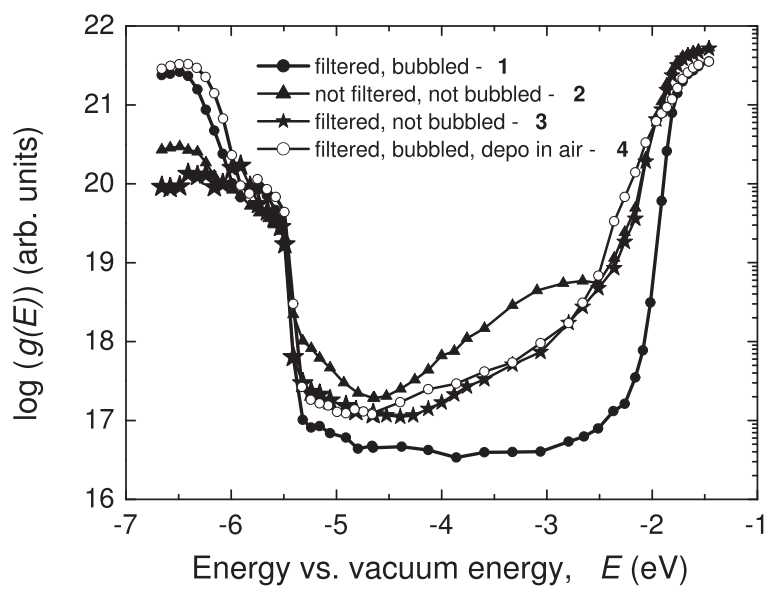

FIG. 2. DOS functions of PMPSi films prepared under various conditions as measured by the ER-EIS method. Line 1, film spin-coated from filtered and 20-min-bubbled solution; line 2, film spin-coated from unfiltered and nonbubbled solution; line 3, film spin-coated from filtered but non-bubbled solution; line 4, film spin-coated and air-dried from filtered and bubbled solution.
DOS of the transport paths. Figure 2 also shows that the absence of filtering and bubbling of the PMPSi solution and spin-coating and drying in the air all introduce extra defect states into the band gap.

Insufficiently dissolved PMPSi aggregates cause defect states between the HOMO and LUMO (line 2). Removing these aggregates by filtration reduces these extra states, but some states spanning up to about $2 \mathrm{eV}$ below LUMO are still present (line 3 ). These are probably caused by oxygen complexes ${ }^{19}$ and can be removed by careful inert gas bubbling before deposition (line 1). This conclusion is supported by the film that was exposed to atmospheric oxygen and moisture during preparation (line 4), where a similar spectrum was obtained. The oxygen-induced defects on the Si backbone of PMPSi are due to the high oxygen affinity, which causes conjugation breaking with high electronegativity inserted between the $\mathrm{Si}-\mathrm{Si} \sigma$ bonded pairs, as indicated by a much smaller bandwidth than the reported value. ${ }^{19}$ Also, the branching point defects that increase dimensionality strongly reduce the main chain exciton $\sigma^{*} \rightarrow \sigma$ photoluminescence at approximately $365 \mathrm{~nm}$, and lead to $\mathrm{WB}^{20}$ In contrast, a broad visible luminescence band situated around $500 \mathrm{~nm}$ increases with branching, and can be explained by formation of organosilane units and prolonged (strained) $\mathrm{Si}-\mathrm{Si} \mathrm{WB} .{ }^{21}$

UV treatment of PMPSi films strongly affects the DOS distribution near the HOMO level without any visible shift in its position, while the LUMO position remains unaffected (Fig. 3). This result is in accordance with the results of our previous studies of this material by post-transient spacecharge-limited-current spectroscopy ${ }^{10}$ (inset in Fig. 3). Specifically, successive increases in the DOS situated above the HOMO transport path were observed following the formation of electronic WB states after UV degradation, which were reconstructed from post-transient spectroscopy ${ }^{11}$ (also inset in Fig. 3). These results demonstrate the capability of the ER-EIS method to map the DOS distribution directly across the whole band gap. The method's sensitivity enables detection of subtle changes in the DOS caused by differences in film preparation or exposure to degradation agents such as ambient air or UV treatments.

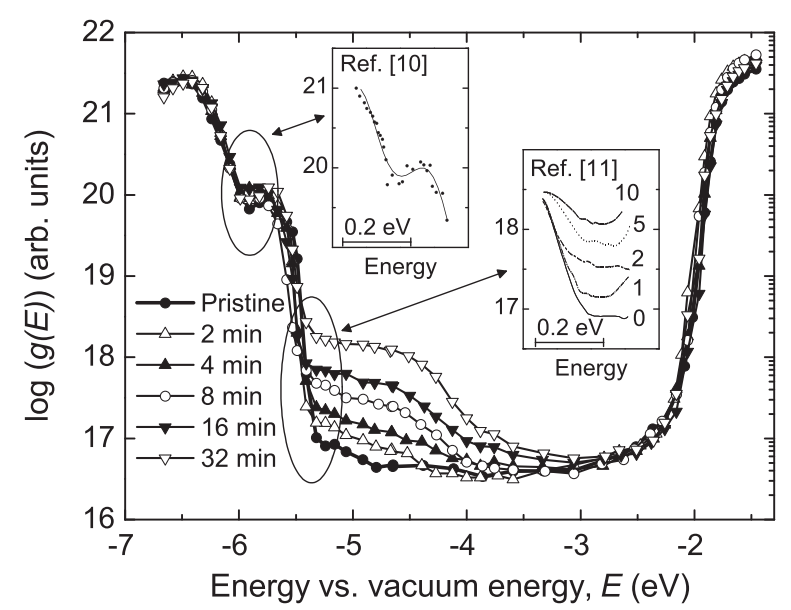

FIG. 3. DOS functions of PMPSi films that were UV-treated for various times, as measured by ER-EIS. For comparison, results obtained by posttransient space-charge-limited-current spectroscopy ${ }^{10}$ and post-transient spectroscopy ${ }^{11}$ are shown in the insets. 
The potential of the ER-EIS method for elucidation of microphysical properties related to the electronic DOS is also demonstrated on PMPSi and two other polymers typically used for molecular electronics, MDMO-PPV, and P3HT, in Fig. 4. The DOS distributions in the linear DOS scale (Fig. 4(a)) were used to evaluate the transport (polaron, bipolaron) band gaps via the methodology used in Ref. 12 . These values are shown at the bottom of the figure. A typical saturation and apparent reduction at the extremes of both the HOMO and LUMO are observed, presumably caused by the filling of the molecular transport states, which reduces both mobility and conductivity. This is in fact observed in the electrochemistry of conducting polymers because of the filling of the finite (Gaussian) DOS. ${ }^{22}$ The same DOS distributions plotted on a log scale (Fig. 4(b)) show all the details of both shallow and deep electronic states with unprecedented sensitivity. Note that this complex picture of the electronic DOS of all three polymers has not previously been published.

The ER-EIS method's sensitivity is also illustrated in Figure 5. The positioning of the HOMO shoulder of MDMO-PPV at $5.2 \mathrm{eV}$ agrees well with published data for $\mathrm{OC}_{1} \mathrm{C}_{10}-\mathrm{PPV}^{5}$ (Fig. 5(a)), which were obtained using an electrochemically-gated transistor. This technique also provided information about DOS over five orders of magnitude, but the defect states in the band gap were not detected because of the limited operating voltage range of the transistor. The ER-EIS spectrum of P3HT is directly compared with its photoemission spectrum, published in Ref. 12, in Figure 5(b). The HOMO onset slope for P3HT was found to be similar to that obtained by photoemission, as shown by overlaying the dependences obtained by both methods (Fig. 5(b)); the photoemission signal proportional to the DOS was aligned with the electrochemical signal according to the HOMO maximum. Photoemission spectroscopy can reconstruct the electronic structure over an energy range of several $\mathrm{eV}$. However, unlike the ER-EIS results, the

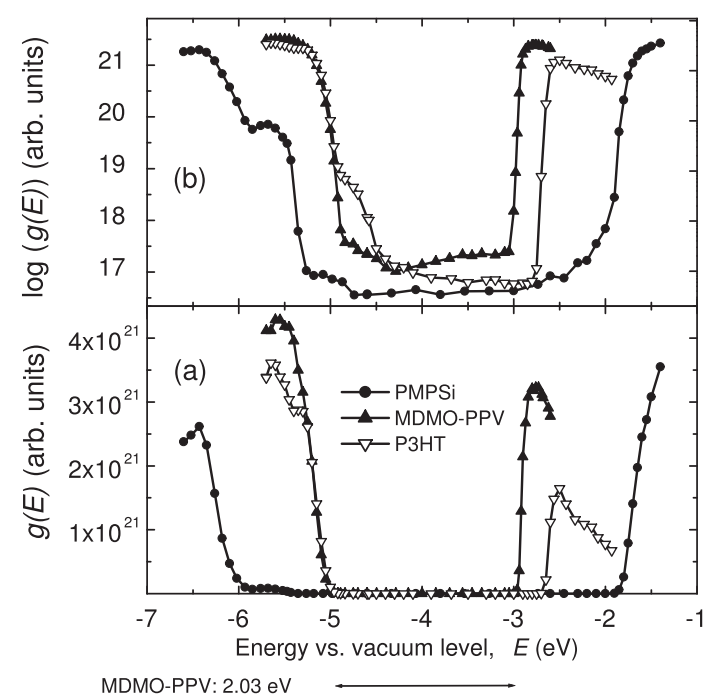

PMPSi: $4.09 \mathrm{eV}$

P3HT: $2.31 \mathrm{eV}$

FIG. 4. DOS functions of PMPSi, MDMO-PPV, and P3HT films as measured by ER-EIS: (a) linear DOS scale, and (b) log DOS scale. The band gaps shown at the bottom are evaluated according to Ref. 12 .

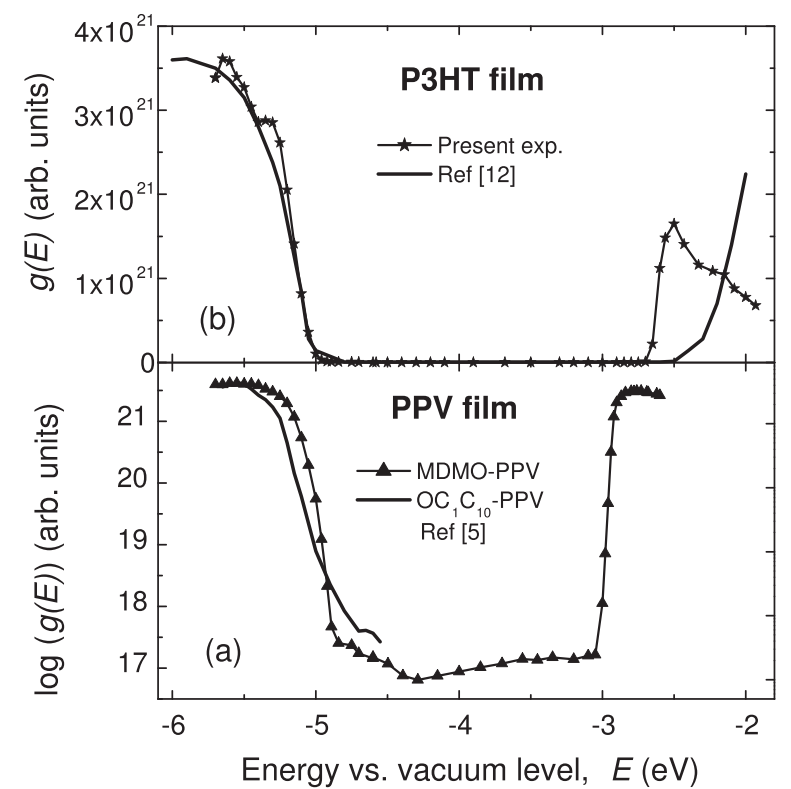

FIG. 5. DOS functions of PPV and P3HT films as measured by ER-EIS and compared with those published in Refs. 5 and 12, respectively.

spectrum lacks information about the fine structure of the DOS in the band gap. The $\log g(E)$ vs $\left(E-E_{\mathrm{o}}\right)^{2}$ plots show that the DOS extremities can be fitted with a Gaussian distribution for both MDMO-PPV $\left(\sigma_{\mathrm{HOMO}}=0.173 \mathrm{eV}\right)$ and P3HT $\left(\sigma_{\text {HOMO }}=0.067 \mathrm{eV}\right)$. The value of $67 \mathrm{meV}$ for P3HT agrees well with the value of $69 \mathrm{meV}$ given in a recent paper $^{23}$ that describes evaluation of time-of-flight transients by an advanced computational procedure.

However, the LUMOs below the extremities of both MDMO-PPV and P3HT show the apparent exponential dependence of the DOS $\left(g(E) \sim \exp \left(E_{0} / E\right)\right)$ with an effective slope of $25 \mathrm{meV}$, corresponding to a measurement temperature of approximately $300 \mathrm{~K}$. This means that the real slope $E_{0}$ for the DOS distributions of the LUMO is lower than $25 \mathrm{meV}$ for both materials.

We can conclude that ER-EIS is a simple and powerful method for mapping of the electronic structures of organic semiconductors. We demonstrated using typical polymers for molecular electronics (PMPSi, MDMO-PPV, P3HT) that it allows DOS mapping over unprecedentedly wide energy and DOS value ranges with energy resolution of $\sim 100 \mathrm{meV}$. In contrast to the competing methods, information about the important DOS parameters is acquired directly from the measured spectra. Also, the method is highly sensitive, and can detect subtle DOS changes caused by modifications to the organic film preparation process or the effects of degradation agents such as ambient air or UV irradiation. These are highly topical issues, e.g., for organic photovoltaic materials. Evaluation of the important DOS parameters, such as the transport band gap, polaron state distributions at the HOMO and LUMO, and the defect state distribution over the entire band gap, was demonstrated using the chosen polymers. The agreement of the measured DOS parameters with the rare previously published data obtained by established methods proves the accuracy of the proposed method. 
The authors would like to acknowledge the financial support of the Slovak Research and Development Agency under Project No. APVV-0096-11, the Scientific Grant Agency VEGA under Project Nos. 2/0157/12 and 2/0041/11, and the partial funding of the KEGA Agency under Project Nos. 011TTU-4/2012 and 020TTU-4/2013.

${ }^{1}$ H. Baessler and A. Koehler, Charge Transport in Organic Semiconductors, Unimolecular and Supramolecular Electronics I, edited by R. M. Metzger (Springer-Verlag, Berlin Heidelberg 2012); Top. Curr. Chem. 312, 1 (2012).

${ }^{2}$ D. D. Macdonald, Electrochim. Acta 51, 1376 (2006).

${ }^{3}$ R. A. Marcus, J. Chem. Phys. 24, 966 (1956); N. S. Lewis, Annu. Rev. Phys. Chem. 42, 543 (1991); A. M. Fajardo and N. S. Lewis, The Spectrum 10 (A Quarterly Publ. Bowling Green State Uni. Ohio, 43403) (Winter, 1997), Vol. 1.

${ }^{4}$ J. Bisquert, F. Fabregat-Santiago, I. Mora-Sero, G. Garcia-Belmonte, E. M. Barea, and E. Palomares, Inorg. Chim. Acta 361, 684 (2008).

${ }^{5}$ I. N. Hulea, H. B. Brom, A. J. Houtepen, D. Vanmaekelbergh, J. J. Kelly, and E. A. Meulenkamp, Phys. Rev. Lett. 93, 166601 (2004).

${ }^{6}$ G. Garcia-Belmonte, J. Bisquert, and G. Popkirov, Appl. Phys. Lett. 83, 2178 (2003).

${ }^{7}$ J. Bisquert, G. Garcia-Belmonte, and J. García-Canadas, J. Chem. Phys. 120, 6726 (2004).
${ }^{8}$ Z. Pomerantz, A. Zaban, S. Ghosh, J.-P. Lellouche, G. Garcia-Belmonte, and J. Bisquert, J. Electroanal. Chem. 614, 49 (2008).

${ }^{9}$ O. Tal, Y. Rosenwaks, Y. Preezant, N. Tessler, C. K. Chan, and A. Kahn, Phys. Rev. Lett. 95, 256405 (2005).

${ }^{10}$ F. Schauer, R. Handlir, and S. Nespurek, Adv. Mater. Opt. Electron. 7, 61 (1997).

${ }^{11}$ F. Schauer, Czech. J. Phys. 49, 871 (1999).

${ }^{12}$ C. Deibel, D. Mack, J. Gorenflot, A. Scholl, S. Krause, F. Reinert, D. Rauh, and V. Dyakonov, Phys. Rev. B 81, 085202 (2010).

${ }^{13}$ N. S. Lewis, J. Phys. Chem. B 102, 4843 (1998).

${ }^{14}$ B. Y. Chang and S. M. Park, Annu. Rev. Anal. Chem. 3, 207 (2010).

${ }^{15}$ See supplementary material at http://dx.doi.org/10.1063/1.4898068 for block diagram of ER-EIS method and energy band diagrams for three (positive, zero, and negative) applied voltages to the sample.

${ }^{16}$ J. O. Oelerich, D. Huemmer, and S. D. Baranovskii, Phys. Rev. Lett. 108, 226403 (2012).

${ }^{17}$ S. Nespurek, J. Non-Cryst. Solids 299-302, 1033 (2002).

${ }^{18}$ J. O. Oelerich, D. Huemmer, M. Weseloh, and S. D. Baranovskii, Appl. Phys. Lett. 97, 143302 (2010).

${ }^{19}$ Y. Sakurai, D. Yoshimura, H. Ishii, Y. Ouchi, H. Isaka, H. Teramae, N. Matsumoto, S. Hasegawa, N. Ueno, and K. Seki, J. Phys. Chem. B 105, 5626 (2001)

${ }^{20}$ W. L. Wilson and T. W. Weidman, J. Phys. Chem. 95, 4568 (1991).

${ }^{21}$ S. Nespurek, F. Schauer, and A. Kadashchuk, Monatsh. Chem. 132, 159 (2001).

${ }^{22}$ J. Bisquert, Phys. Chem. Chem. Phys. 10, 3175 (2008).

${ }^{23}$ J. Lorrmann, M. Ruf, D. Vocke, V. Dyakonov, and C. Deibel, J. Appl. Phys. 115, 183702 (2014). 
Applied Physics Letters is copyrighted by the American Institute of Physics (AIP).

Redistribution of journal material is subject to the AIP online journal license and/or AIP copyright. For more information, see http://ojps.aip.org/aplo/aplcr.jsp 